\title{
APLICAÇÃO DA RESISTIVIDADE E CARGABILIDADE EM ESTUDOS DE CONTAMINAÇÃO DE SEDIMENTOS POR DERIVADOS DE HIDROCARBONETOS
}

\author{
Antonio Celso de Oliveira Braga ${ }^{1}$ e Maíra Tozzi Cardinali² \\ Recebido em 04 abril, 2005 / Aceito em 23 setembro, 2005 \\ Received on April 04, 2005 / Accepted on September 23, 2005
}

\begin{abstract}
This paper describes the results obtained with DC resistivity and time domain induced polarization (IP) electrical methods, through the vertical electrical sounding (Schlumberger array) and profiling (dipole-dipole and gradient arrays) techniques. This study was conducted in reduced models, with in tanks containing claysand layer and sand layer sediments of the Rio Claro Formation, contaminated with gasoline. Our results demonstrated that the resistivity of the sediments increased significantly, immediately after the contamination, but their chargeability decreased sensibly. The contaminated soils were monitored for ten months, being observed that after six months, the resistivity of the superficial materials became lower, in relation to the readings without contamination, while the chargeability stayed drops. The obtained data were interpreted in a qualitative and quantitative by using 1-D and 2-D models.
\end{abstract}

Keywords: Resistivity, chargeability, hydrocarbon derived, contamination.

RESUMO. Esse trabalho descreve os resultados obtidos com os métodos da eletrorresistividade e polarização induzida, usando as técnicas de campo da sondagem elétrica vertical (arranjo Schlumberger) e caminhamento elétrico (arranjos dipolo-dipolo e gradiente). Os estudos foram efetuados em modelos reduzidos, em tanques contendo sedimentos areno-argilosos e arenosos da Formação Rio Claro, contaminados por derivados de hidrocarbonetos (gasolina). Os ensaios demonstraram que a resistividade dos sedimentos aumentou significativamente, imediatamente após a contaminação, enquanto que a cargabilidade diminuiu sensivelmente. As contaminações foram monitoradas durante dez meses, sendo observado que após seis meses, a resistividade dos materiais superficiais tornou-se menor, em relação às leituras sem contaminação, enquanto que as cargabilidades mantiveram-se baixas. Os dados obtidos foram interpretados qualitativamente e quantitativamente (1-D e 2-D).

Palavras-chave: Resistividade, cargabilidade, derivados de hidrocarbonetos, contaminação.

\footnotetext{
1 IGCE-UNESP - Departamento de Geologia Aplicada, Av. 24-A, n 1515 - CEP 13500-230 Rio Claro, SP, Brasil. Tel: (19) 3526-2803; Fax: (19) 3534-0327 -E-mail: acobraga@rc.unesp.br

${ }^{2}$ Mestranda - Curso de Pós Graduação em Geociências - IGCE-UNESP - Departamento de Geologia Aplicada, Av. 24-A, n 1515 - CEP 13500-230 Rio Claro, SP, Brasil. Tel: (19) 3526-2803; Fax: (19) 3534-0327 - E-mail: maira_cardi@yahoo.com.br
} 


\section{INTRODUÇÃO}

Este trabalho descreve os resultados obtidos com os métodos da eletrorresistividade e polarização induzida, através das técnicas de campo da sondagem elétrica vertical - arranjo Schlumberger e caminhamento elétrico - arranjos dipolo-dipolo e gradiente, desenvolvidos em modelo reduzido. 0 objetivo foi o de efetuar um estudo das variações da resistividade e cargabilidade de materiais arenosos e areno-argilosos, quando saturados por contaminantes derivados de hidrocarbonetos (Cummings, 1990; Braga, 2001).

0 estudo realizou-se em escala reduzida de campo, procurando determinar um padrão de variabilidade nas propriedades elétricas, que poderia ser utilizado em estudos reais de contaminação dos solos e águas subterrâneas. As medidas da resistividade e cargabilidade foram efetuadas, com o material não saturado e saturado com água, obtendo uma caracterização em função do grau de saturação. Posteriormente, com a introdução dos contaminantes, as propriedades elétricas foram analisadas temporalmente, procurando identificar suas variações em função das reações entre o meio geológico e o material contaminante.

Segundo Sauck (2000), contaminantes derivados de hidrocarbonetos, apresentam valores elevados de resistividade; entretanto, estes valores variam com 0 tempo, em função de reações com ácidos orgânicos ou ácidos carbônicos devido a biodegradação dos componentes do hidrocarboneto, tornandoos condutivos. Em estudos recentes, executados pelo Departamento de Geologia Aplicada-IGCE em refinarias de combustíveis da Petrobrás, os valores obtidos para estes contaminantes foram baixos, provavelmente, indicando antigos vazamentos.

\section{METODOLOGIA E AQUISIÇÃO DOS DADOS Laboratório}

Os estudos foram executados em um tanque de fibra de vidro, com dimensões $98 \times 40 \times 33 \mathrm{~cm}$ (Figura 1). 0 tanque foi preenchido com sedimentos arenosos (arenito da Fm Rio Claro), arenoargilosos (solo da Fm Rio Claro), tendo na base, brita. Na lateral do tanque, foi acoplado um piezômetro, com a finalidade de controlar o nivel d'água. Foram ainda, escavadas duas trincheiras laterais $(5 \times 5 \times 8 \mathrm{~cm})$, nas extremidades, visando simular áreas de recarga das águas subterrâneas.

Quanto à contaminação, parte da gasolina foi introduzida em quatro pequenos furos de $6 \mathrm{~cm}$ de profundidade e $0,5 \mathrm{~cm}$ de diâmetro, e parte derramada na superfície, sendo utilizados 2 litros de contaminantes, simulando um vazamento. Os espaçamentos dos eletrodos foram programados procurando obedecer ao fator escala 100. Os eletrodos de corrente e de potencial, foram confeccionados em alumínio com dimensões de $1,5 \mathrm{~cm}$ de comprimento e $0,1 \mathrm{~cm}$ de diâmetro. Apparao et al. (1997), Goes \& Meekes (2004), discutem as relações espaçamento/profundidade de investigação em materiais resistivos, analisando a intensidade e localização das anomalias obtidas, os quais auxiliaram na escolha dos espaçamentos.

Os ensaios geofísicos foram realizados no período de janeiro a outubro de 2004 (dez meses), sendo executadas um total de 15 sondagens elétricas verticais - Schlumberger (SEV), 11 caminhamentos elétricos - Dipolo-Dipolo (CE-DD) e 13 caminhamentos elétricos - Gradiente (CE-GRAD).

\section{Sondagem Elétrica Vertical - SEV}

Nas SEV's, cujos procedimentos gerais são apresentados por Orellana (1972), Robinson \& Çoruh (1988) e Draskovits et al. (1990), utilizou-se do espaçamento máximo AB/2 de $16 \mathrm{~cm}, 0$ qual considerou a menor dimensão da caixa utilizada, procurando evitar possível efeito lateral das paredes do tanque, nas leituras obtidas. 0 modelo resultante, resistividade e cargabilidade (Oldenburg \& Li, 1994; Ward, 1990), da interpretação das SEV's, foi obtido pelo software IX1D V. 2 (Interpex Limited-USA).

\section{Caminhamento Elétrico - CE}

Para 0 CE - arranjo dipolo-dipolo, o espaçamento utilizado foi de $5 \mathrm{~cm}$. Com quatro níveis de investigação, com as seguintes profundidades teóricas: $2,5-3,75-5,0-6,25 \mathrm{~cm}$. Para 0 dipolo-dipolo, foi locada uma linha de $50 \mathrm{~cm}$. No arranjo Gradiente, os espaçamentos foram os seguintes: $A B=40 \mathrm{~cm} ; \mathrm{MN}=$ $2 \mathrm{~cm}$; correspondente a uma profundidade teórica de investigação de $10 \mathrm{~cm}$. A área central investigada foi de $14 \times 16 \mathrm{~cm}$, na qual foram locadas 9 linhas, de $14 \mathrm{~cm}$ cada, espaçadas entre si de $2 \mathrm{~cm}$.

A interpretação dos dados obtidos no CE foi efetuada qualitativamente, usando perfis e mapas de valores aparentes. Posteriormente os dados do CE-DD, foram invertidos através da inversão pelo software Res2dinv V. 3.5 (Geotomo Software - Malaysia).

\section{Equipamento Geofísico}

0 equipamento geofísico utilizado foi o sistema IP-resistividade digital da ABEM - SAS 4000/Suécia, composto de um módulo (receptor e transmissor), pertencente ao Departamento de Geologia Aplicada - IGCE da UNESP. Esse equipamento revelou-se de grande precisão na obtenção dos dados, onde devido ao caráter de ensaios em escala reduzida, a gama de variação na corrente 


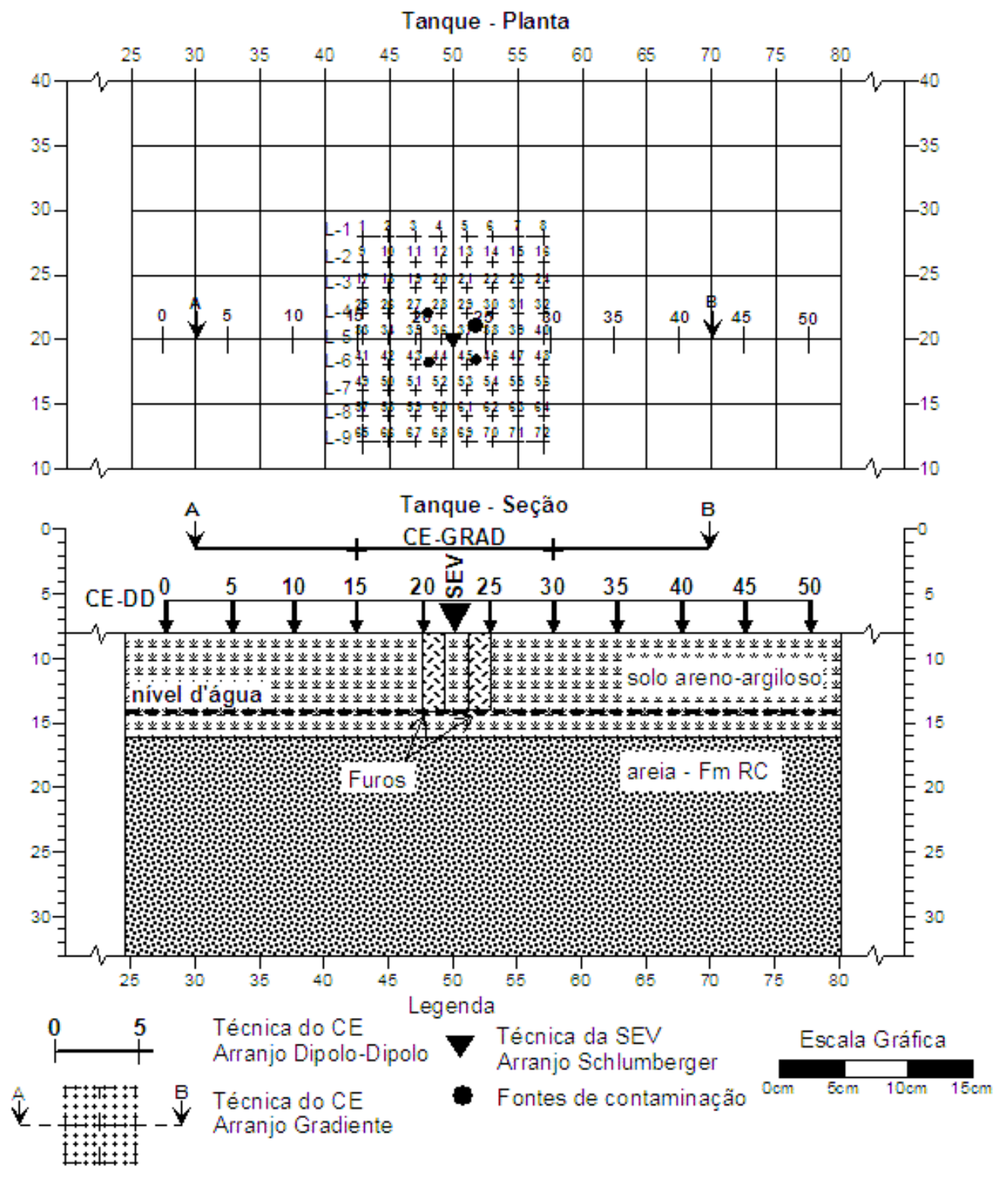

Figura 1 - Tanque e localização dos estudos geofísicos.

Tabela 1 - Planejamento de início dos estudos geofísicos.

\begin{tabular}{|c|l|l|}
\hline Data & \multicolumn{1}{|c|}{ Técnica/arranjo } & \multicolumn{1}{c|}{ Situação } \\
\hline 05/01/04 & Sondagem Elétrica Vertical & - sem gasolina. \\
\hline 06/01/04 & Caminhamento Gradiente & $\begin{array}{l}\text { - primeira leitura, sem gasolina. } \\
\text { - segunda leitura, com gasolina - 30 minutos } \\
\text { depois de contaminado. }\end{array}$ \\
\hline 06/01/04 & Caminhamento Dipolo-Dipolo & - sem gasolina. \\
\hline 06/01/04 & Sondagem Elétrica Vertical & - 60 minutos após contaminação de gasolina. \\
\hline 07/01/04 & Caminhamento Dipolo-Dipolo & - com gasolina. \\
\hline
\end{tabular}


elétrica/potencial, necessitava ser compatível com os ensaios realizados.

\section{DISCUSSÃO DOS RESULTADOS}

Os ensaios geofísicos foram desenvolvidos, inicialmente, conforme Tabela 1.

\section{Caminhamento Elétrico - Dipolo-Dipolo}

A Figura 2 apresenta as pseudo-seções de resistividade aparente e cargabilidade aparente nos ensaios:

- DD-1 (06/01) - sem contaminação;

- DD-2 (07/01) - um dia após a contaminação; e,

- DD-10 (05/08) - 212 dias após a contaminação.

0 ensaio de 06/01 (DD-1), não apresentou variações significativas nos valores de resistividade aparente, quanto a cargabilidade aparente, a partir da estaca 25, os níveis mais superficiais, apresentaram valores mais elevados do que no resto da seção. Já, os resultados do dia 07/01 (DD-2), apresentaram uma anomalia de altos valores de resistividade aparente, com dois flancos perfeitamente definidos; enquanto que os valores de cargabilidade aparente, mostraram uma anomalia de baixos valores, mais intensa no flanco direito (efeito de eletrodos de corrente). As anomalias identificadas no DD-2 (07/01) foram projetadas na superfície do tanque, entre as estacas 20 e 25, correspondendo 0 local da fonte de contaminação.

No ensaio DD-10 (05/08), após 212 dias da contaminação. Pode-se observar, que a resistividade aparente apresentou valores bem baixos, em relação ao início da contaminação. Já, a cargabilidade aparente, continuou com valores baixos.

A Figura 3 apresenta o modelo geoelétrico dos ensaios DD1 (06/01), DD-2 (07/01) e DD-10 (05/08), após a inversão dos dados das pseudo-seções de resistividade aparente.

Observa-se que o modelo da resistividade após a inversão de DD-1 (06/01), definiu, uma faixa mais condutiva, por volta da profundidade de $6 \mathrm{~cm}$, a qual corresponde ao nível d'água. Acima, podem-se separar as prováveis faixas existentes na zona não saturada: faixa de umidade (evapotranspiração), sem umidade (retenção) e capilar.

0 modelo de resistividade, resultante da inversão do DD-2 (07/01), definiu perfeitamente a posição da pluma de contaminação, apresentando altos valores de resistividade, entre as estacas 20-25, estendendo verticalmente até aproximadamente $4,5 \mathrm{~cm}$ de profundidade. Pode-se observar, também, que esta pluma, apresenta, em profundidade, uma extensão lateral, até aproximadamente a estaca 35. Esse fluxo do contaminante, pode confirmar a hipótese anterior, sobre um aumento da porcentagem de sedimentos arenosos neste trecho investigado.

Quanto ao ensaio DD-10 (05/08), o modelo de resistividade, demonstra que, após 212 dias da contaminação, os valores tornaram-se condutivos em relação ao modelo inicial de DD-1 (06/01), apresentando um deslocamento para as estacas 25-30.

A Figura 4 apresenta o modelo geoelétrico dos ensaios DD1 (06/01), DD-2 (07/01) e DD-10 (05/08), após a inversão dos dados das pseudo-seções de cargabilidade aparente.

0 modelo resultante de DD-1 (06/01), como visualizado qualitativamente nas pseudo-seções, definiu valores mais elevados a partir da estaca 25 - primeiros níveis. Como 0 solo utilizado é argilo-arenoso, esse aumento nos valores de cargabilidade associado a um aumento nos valores de resistividade, pode estar refletindo uma maior porcentagem de sedimentos arenosos.

0 parâmetro cargabilidade de DD-2 (07/01), apresentou valores mais elevados entre as estacas $30 \mathrm{e} 35$. Entre as estacas 20 e 25, os valores tornaram-se baixos, refletindo uma diminuição da cargabilidade dos sedimentos, quando contaminados. Para os valores de cargabilidade de DD-10 (05/08), observa-se que pouco mudou em relação ao ensaio anterior.

\section{Caminhamento Elétrico - Gradiente}

A Figura 5 apresenta os resultados obtidos pelo CE-Gradiente, respectivamente, antes da contaminação (GRAD-1, dia 06/01), um dia após (GRAD-3, dia 07/01) e 252 dias após (GRAD-13, dia 15/09) a contaminação por gasolina. Quanto à resistividade aparente, observa-se que no ensaio executado um dia após a contaminação (GRAD-3), os valores foram mais elevados, ao redor dos furos, do que no ensaio GRAD-1.

Os valores do GRAD-13 (15/09), apresentaram valores bem mais baixos que 0 GRAD-1, refletindo uma maior condutividade após 252 dias da contaminação.

Quanto aos valores de cargabilidade aparente, nessas mesmas figuras, as variações não foram bem definidas, apresentando no GRAD-2 (07/01), de um modo geral, valores mais elevados que no GRAD-1 (06/01), e posteriormente, valores mais baixos. As leituras do GRAD-13 (15/09), ainda apresentam valores baixos. Nesse arranjo, pode-se concluir que a cargabilidade respondeu à contaminação. Ressalta-se, como comentado anteriormente, a profundidade teórica de investigação do gradiente, foi de $10 \mathrm{~m}$. 


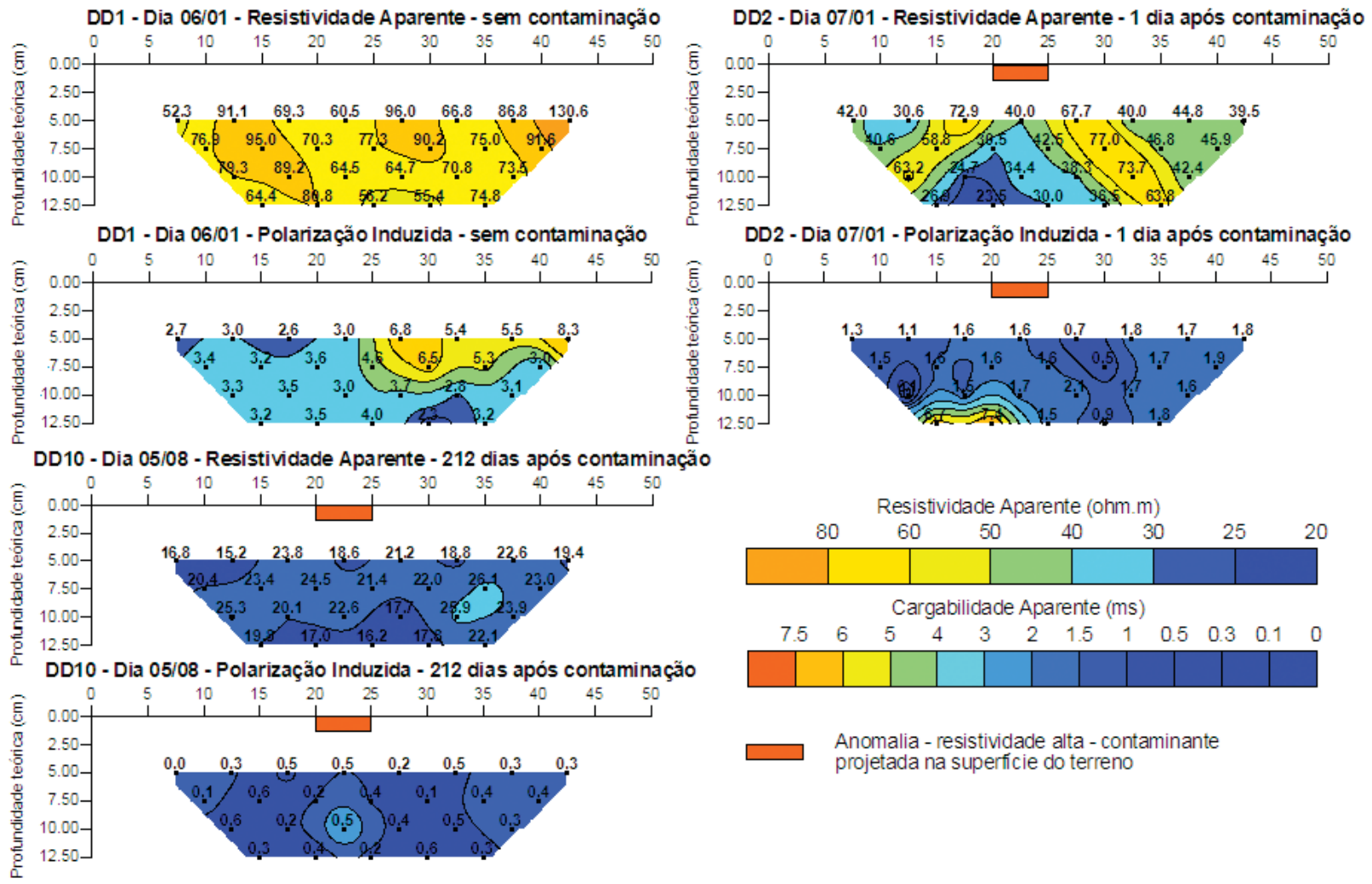

Figura 2 - Dipolo-Dipolo DD-1 (06/01), DD-2 (07/01) e DD-10 (05/08).

\section{Sondagem Elétrica Vertical - Schlumberger}

Para as SEV's, a análise dos resultados foi efetuada qualitativa e quantitativamente. Foram selecionadas algumas SEV's representativas para efeito de apresentação nesse trabalho.

- SEV-1 (05/01) - sem contaminação;

- SEV-2 (06/01) - 60 minutos após a contaminação;

- SEV-6 (04/02) - 29 dias após a contaminação;

- SEV-9 (03/03) - 57 dias após a contaminação;

- SEV-11 (22/04) - 107 dias após a contaminação;

- SEV-13 (12/08) - 219 dias após a contaminação; e,

- SEV-15 (08/10) - 275 dias após a contaminação.

$\mathrm{Na}$ análise qualitativa, observa-se que as curvas de campo (Figura 6), resistividade e cargabilidade aparentes, apresentaram alterações significativas, variando totalmente de década na escala log (eixo y), considerando a SEV-01 (05/01) - sem gasolina, e
SEV-02 (06/01) - com gasolina. Com a contaminação, os valores de resistividade aumentaram expressivamente e os valores de cargabilidade diminuíram.

$\mathrm{Na}$ resistividade, as curvas de campo apresentaram pequenas variações para os espaçamentos iniciais, refletindo, provavelmente, alterações de saturação. Nos espaçamentos finais, a partir do $\mathrm{AB} / 2=10 \mathrm{~cm}$, os ramos são praticamente coincidentes. As SEV's dos meses 08 e 10, a partir de 219 dias após a contaminação, apresentam para os espaçamentos iniciais, valores de resistividades menores que a SEV-01 (05/01) - sem contaminação.

$\mathrm{Na}$ cargabilidade, as curvas apresentam variações que podem ser associadas, também, a variações de saturação. Entretanto, as SEV's dos meses 08 e 10, diferentes da resistividade, mantiveram as faixas de variação após a contaminação. Com destaque apenas para a SEV-15 (08/10), que apesar de manter os valores ainda abaixo da SEV-01 (05/01), apresenta uma forma quase semelhante.

As Figuras 7 e 8 foram construídas procurando quantificar as variações nos valores, respectivamente, de resistividade e cargabilidade, de cada SEV - pós-contaminação, considerando cada 


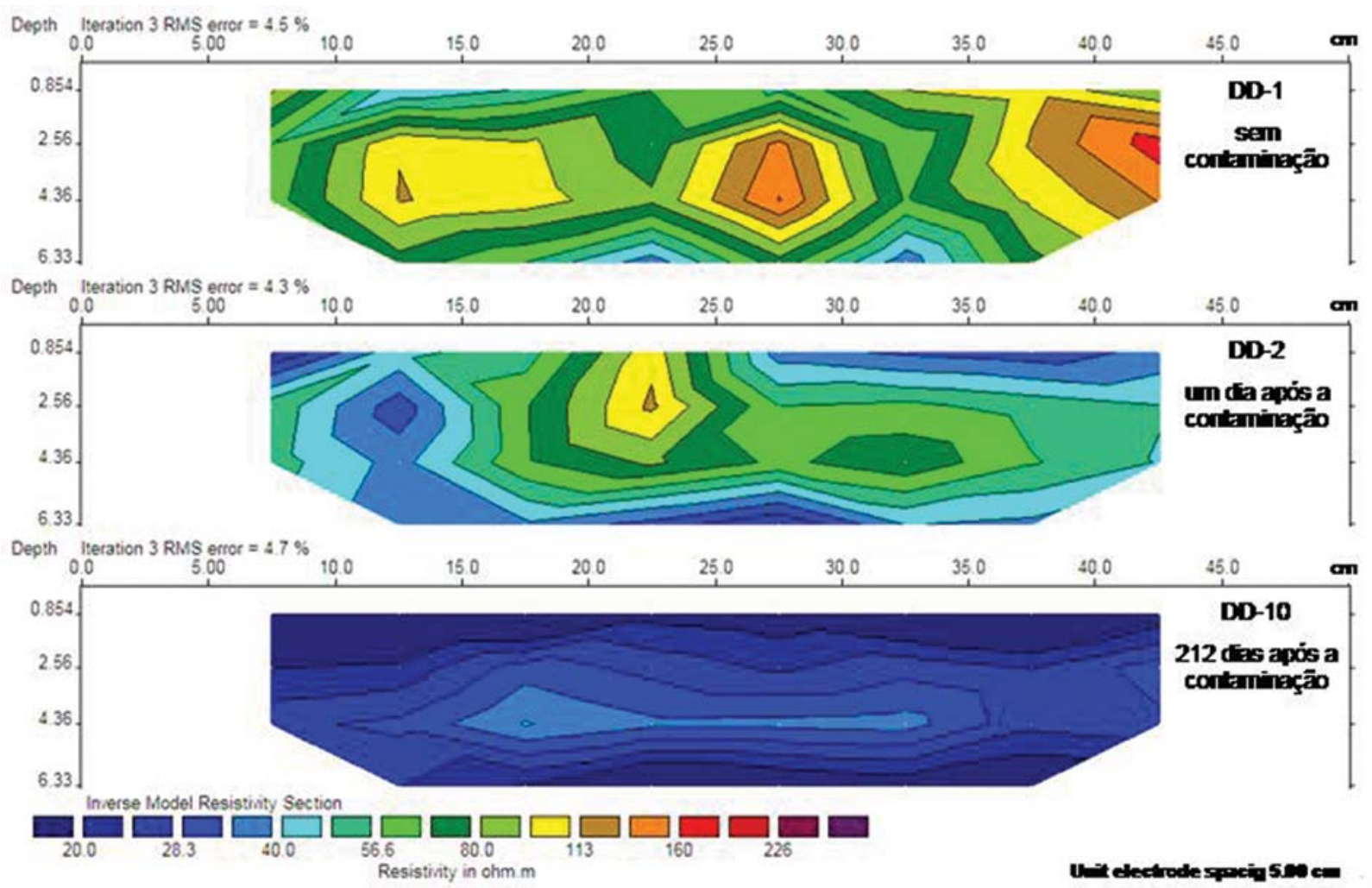

Figura 3 - Resistividade DD-1 (06/01), DD-2 (07/01) e DD-10 (05/08) - Inversão/Res2dinv.

espaçamento AB/2 utilizado, em relação às leituras da SEV-01 pré-contaminação.

Os valores de resistividade (Figura 7), entre os ensaios do dia 05/01 (SEV-01) e 06/01 (SEV-02), apresentaram variação média de, aproximadamente, 4 vezes. Isso mostra que, imediatamente após a contaminação, os sedimentos tornam-se mais resistivos, conforme já constatado nos ensaios do CE. Posteriormente, essa variação diminui, tendendo a 1. As SEV-13 (12/08) e 15 (08/10), executadas a partir de sete meses da contaminação, apresentaram, principalmente nas profundidades mais rasas, valores menor que 1 , evidenciando que o meio estudado, torna-se condutivo com 0 passar do tempo.

Para os valores de cargabilidade aparente (Figura 7), a variação também foi significativa, com valores mais baixos que os iniciais, sem contaminação. Entretanto, com o tempo, os valores se tornaram, aproximadamente, constantes, diferentemente da resistividade. Entretanto a SEV-15 (08/10), tende a apresentar uma relação superior, mais próxima a 1. Em todos os ensaios, pode-se observar que a cargabilidade "demora" mais que a resistividade, em responder ao contaminante, quer mais resistivo quer mais condutivo.
$\mathrm{Na}$ interpretação quantitativa das SEV's, primeiramente, definiu-se o modelo da SEV-01 (05/01), não contaminada. Posteriormente, após a contaminação com gasolina, o modelo foi ajustado, a partir deste ensaio, considerando a introdução do contaminante.

Outro fato a comentar, diz respeito à profundidade do nível d'água (NA), o qual durante todos os ensaios, foi programado para manter, aproximadamente, $6 \mathrm{~cm}$. Apesar de esperarem-se variações normais na profundidade do NA, a presença do contaminante, mascarou totalmente sua posição, a partir da SEV-06 (04/02). Entretanto, pode-se afirmar que o NA nas demais SEV's, corresponderia ao topo da camada mais resistiva em profundidade (areia da Formação Rio Claro). Por outro lado, as profundidades mais rasas do topo das areias da Fm RC, poderiam ser, provavelmente, função de uma diminuição da espessura dos sedimentos superficiais, devido a uma maior compactação com 0 tempo.

Essas imprecisões na determinação do NA, mostra a dificuldade em determinar-se com precisão a profundidade do NA em estudos ambientais, onde 0 contaminante altera as 


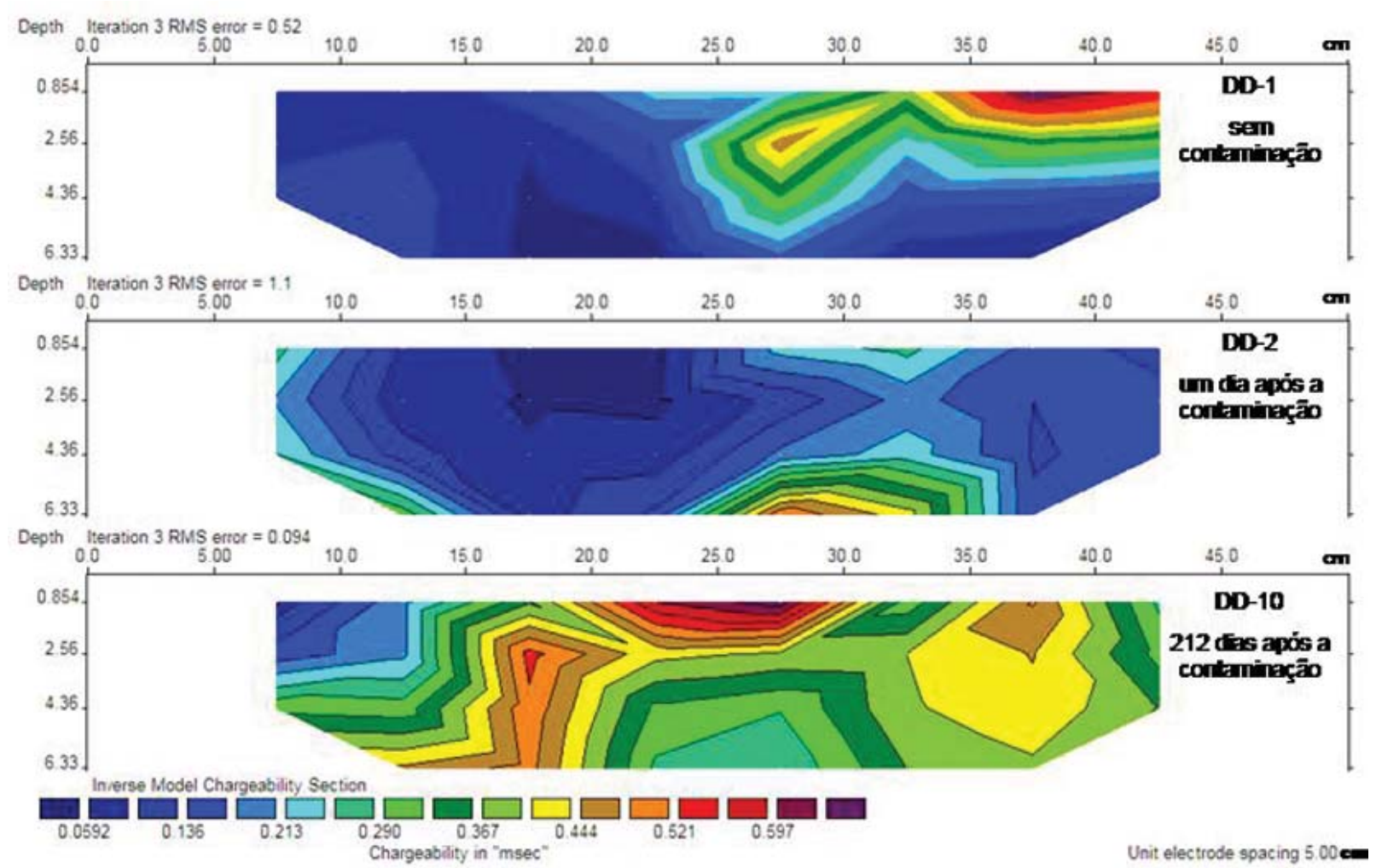

Figura 4 - Cargabilidade DD-1 (06/01), DD-2 (07/01) e DD-10 (05/08) - Inversão/Res2dinv.

resistividades dos materiais geológicos naturais, normalmente para valores baixos.

\section{CONCLUSÕES}

Os ensaios geofísicos executados, através dos métodos da eletrorresistividade e polarização induzida, técnicas de campo da sondagem elétrica vertical (Schlumberger) e caminhamento elétrico (Dipolo-Dipolo e Gradiente), identificaram perfeitamente o contaminante - gasolina.

Enquanto que a resistividade dos sedimentos aumentou significativamente, imediatamente após a introdução do contaminante, a cargabilidade diminuiu. Com o passar do tempo, aproximadamente seis meses após a contaminação, os valores de resistividade diminuíram, tornando os sedimentos mais condutivos em relação ao meio não contaminado. A cargabilidade, apesar de não voltar ao padrão inicial, apresentou uma tendência de o fazer.

Portanto, pode-se concluir de uma maneira geral, que em vazamentos de derivados de hidrocarbonetos, atingindo os solos e rochas sedimentares e as águas subterrâneas, o fator tempo é fundamental. Em relação ao meio natural (sem contaminante), vaza- mentos recentes deverão apresentar: resistividades altas - anomalias resistivas, e cargabilidades baixas - anomalias de baixa cargabilidade. Em vazamentos antigos, a situação se inverte: a resistividade deverá ser baixa - anomalias condutivas, e a cargabilidade alta - anomalias de alta cargabilidade.

Portanto, em áreas contaminadas, as resistividades naturais da zona não saturada, normalmente, diminuem devido à presença de determinados tipos de contaminantes, por exemplo: chorumes, dificultando a correta definição do nível d'água.

\section{AGRADECIMENTOS}

A Fundação de Apoio à Pesquisa do Estado de São Paulo FAPESP, pelo apoio financeiro da pesquisa, ao Departamento de Geologia Aplicada - IGCE/Unesp, pelo apoio técnico e instrumental, e ao Programa de Formação de Recursos Humanos em Geologia e Ciências Aplicadas ao Setor de Petróleo e Gás - Convênio ANP/DGA-IGCE/Unesp, pela bolsa de Iniciação Científica para aluno envolvido na pesquisa. 


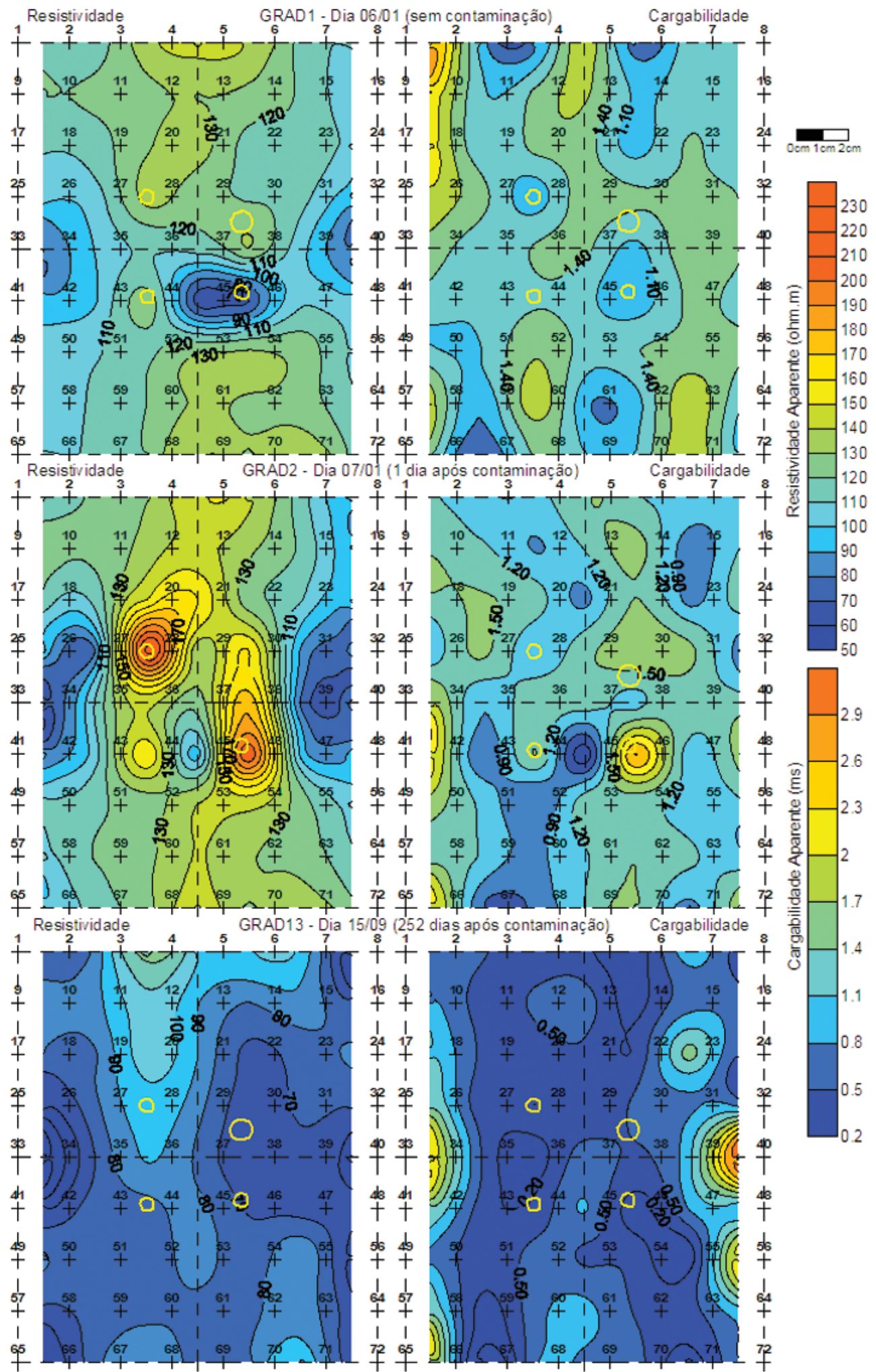

Figura 5 - Caminhamento GRAD-1 (06/01) - GRAD-3 (07/01) - GRAD-13 (15/09). 

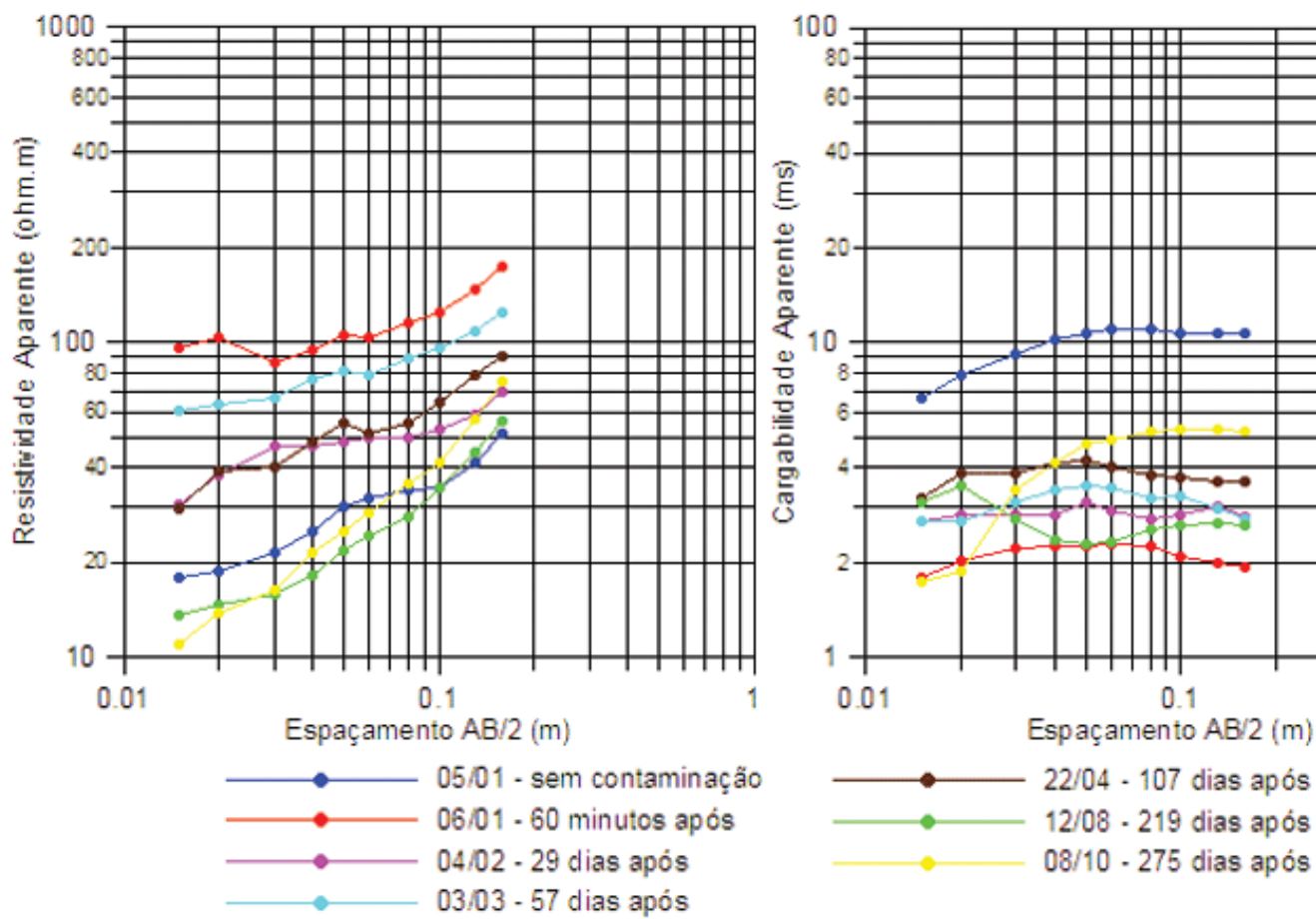

Figura 6 - SEV/curvas de campo - Resistividade e Cargabilidade.
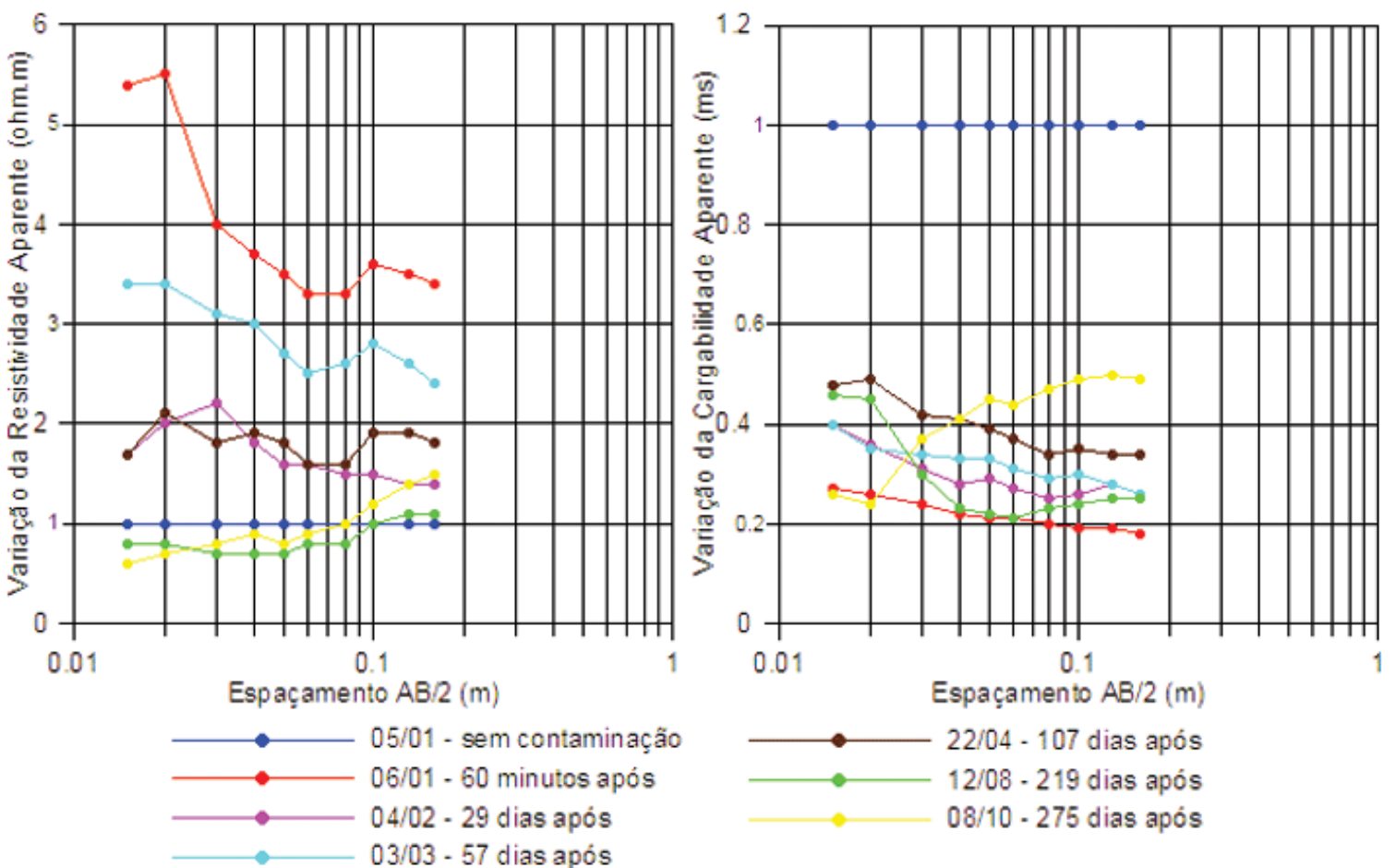

Figura 7 - Variação das resistividades e cargabilidades em relação a SEV-01 (05/jan). 


\section{REFERÊNCIAS}

APPARAO A, SRINIVAS S \& SARMA VS. 1997. Physical modeling results on modified pseudodepht sections in exploration of Highly Resistive Targets; Pure Applied Geophysics, ISSN: 0033-4553 (Paper) 1420-9136 (Online), Issue: 150(2) 341-352.

BRAGA AC0. 2001. "Métodos Geoelétricos Aplicados no Diagnóstico do Lençol Freático para Subsídio em Estudos Ambientais". Projeto de Pesquisa - Bolsa de Produtividade em Pesquisa (PQ). Entidade Financiadora: Conselho Nacional de Desenvolvimento Científico e Tecnológico - CNPq, Coordenação de Ciências Exatas e da Terra 2 - Programa Básico de Geociências: Geofísica, Meteorologia e Geodésia. Período: 01/08/2000 a 31/07/2002.

CUMMINGS D. 1990. Surface geophysical investigations for Hazardous Waste Sites. Geophysical Applications for Geotechmical Investigations, ASTM STP 1101, Frederick L.P. and Wayne R.S. Eds., American Society for Testing and Materials, Philadelphia, p. 9-16.

DRASKOVITS P, HOBOT J, VERÖ L, SMITH B. 1990. Inducedpolarization surveys applied to evaluation of groundwater resources, Pannonian Basin, Hungary. USA. Investigations in Geophysics, $n^{\circ} 4$. Induced Polarization Applications and Case Histories. Society of Exploration Geophysicists, Ed.Stanley H. Ward, p. 379-396.

FERREIRA J \& ZUQUETTE LV. 1998. Considerações sobre as interações entre contaminantes constituídos de hidrocarbonetos e os componentes do meio físico. Geociências, São Paulo, 17(2): 527-557.
GEOTOMO SOFTWARE Res2dinv V. 3.5 -2D Resistivity and IP Inversion, Malaysia.

GOES BJM \& MEEKES JAC. 2004. An effective electrode configuration for detection of DNAPLs with electrical resistivity tomography. Journal of Environmental \& Engineering Geophysics, 9(3): 127-141.

INTERPEX LIMITED SOFTWARE IX1 V. 2.0 - 1D Sounding Inversion, USA.

OLDENBURG DW \& LI Y. 1994. Inversion of induced polarization data. Geophysics, 59(9): 1327-1341.

ORELLANA E. 1972. Prospeccion geoelectrica en corriente continua. Madrid, Ed. Paraninfo, Biblioteca Tecnica Philips, 523 p.

ROBINSON ES \& ÇORUH C. 1988. Basic exploration geophysics. EUA, Ed. John Wiley Sons, Inc., Cap. 13 - Geoelectrical Surveying, p. 445500.

SAUCK WA. 2000. A model for the resistivity structure of LNAPL plumes and their environs in sandy sediments. Journal of Applied Gephysics, 44. Elsevier Scientific Publishing Company, Amsterdam, p. 151-165.

WARD SH. 1990. Resistivity and induced polarization methods. USA. Investigations in Geophysics, $n^{\circ}$ 5. Geotechnical and Environmental Geophysics. Society of Exploration Geophysicists, Ed.Stanley H. Ward, v. I, p. 147-189.

\section{NOTAS SOBRE OS AUTORES}

Antonio Celso de Oliveira Braga é geólogo pela Universidade Estadual Paulista-UNESP/Campus de Rio Claro (1978). Doutorado em Geociências pela Universidade Estadual Paulista-UNESP/Campus de Rio Claro (1977). Trabalhou no Instituto de Pesquisas Tecnológicas do Estado de São Paulo-IPT (1978 a 1995). Desde 1998 é Professor Doutor do Departamento de Geologia Aplicada-IGCE/UNESP - Rio Claro, atuando com geofísica aplicada à hidrogeologia e estudos ambientais, envolvendo os métodos geoelétricos. De 2002 a 2004, foi vice-coordenador do Programa de Pós-Graduação em Geociências - Área de Concentração em Geociências e Meio Ambiente-IGCE/UNESP

Maíra Tozzi Cardinali é geóloga pela Universidade Estadual Paulista-UNESP/Campus de Rio Claro (2005). Mestrado em Geociências pela Universidade Estadual Paulista-UNESP/Campus de Rio Claro (em andamento). De 2002 a 2003, foi bolsista do CNPq - PIBIC - Bolsa de Iniciação Científica. Em 2004, foi bolsista pelo Programa de Formação de Recursos Humanos em Geologia e Ciências Aplicadas ao Setor de Petróleo e Gás - Convênio ANP/DGA-IGCE/Unesp. 